Research Paper

\title{
Glucocorticoid Regulates NLRP3 in Acute-On-Chronic Hepatitis B Liver Failure
}

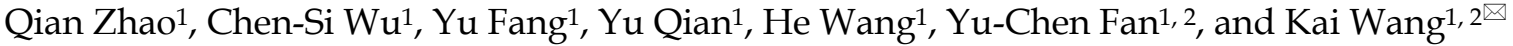 \\ 1. Department of Hepatology, Qilu Hospital of Shandong University, Jinan 250012, China \\ 2. Institute of Hepatology, Shandong University, Jinan 250012, China \\ $\square$ Corresponding author: Kai Wang, MD, PhD, Department of Hepatology, Qilu Hospital of Shandong University and Hepatology Institute of Shandong \\ University, Wenhuaxi Road 107\#, Jinan 250012, Shandong, China. Email: wangdoc876@126.com; wangdoc2010@163.com. Tel: +86-531-82169593; Fax: \\ $+86-531-86927544$ \\ (c) Ivyspring International Publisher. This is an open access article distributed under the terms of the Creative Commons Attribution (CC BY-NC) license \\ (https:// creativecommons.org/licenses/by-nc/4.0/). See http://ivyspring.com/terms for full terms and conditions.
}

Received: 2018.10.04; Accepted: 2019.02.12; Published: 2019.03.09

\begin{abstract}
Acute-on-chronic hepatitis $B$ liver failure (ACHBLF) refers to the acute deterioration of liver function during chronic hepatitis $B$ virus infection, and is associated with high mortality, with rapid progression to death. Nucleotide-binding oligomerisation domain-like receptors (NLRs) Family Pyrin Domain Containing 3(NLRP3) inflammasome contributed to the pathogenesis of D-galactosamine and lipopolysaccharide-induced acute liver failure. However, the profile of NLRP3 in patients with ACHBLF has not been demonstrated. This study was therefore conducted to investigate the expression of NLRP3 in patients with ACHBLF and identify the effect of glucocorticoid on NLRP3. We recruited 70 patients with ACHBLF undergoing glucocorticoid treatment for 28 days, 30 patients with chronic hepatitis B (CHB), and 24 healthy controls (HCs) in this study. The relative messenger RNA (mRNA) level of NLRP3 and related genes were measured by reverse transcription polymerase chain reaction, the plasma levels of interleukin-1 $\beta$ (IL-1 $\beta$ ) and interleukin-18 (IL-18) were measured by enzyme-linked immunosorbent assay. The mRNA level of NLRP3 was significantly higher in patients with $A C H B L F$ than in patients with $\mathrm{CHB}$ as well as $\mathrm{HCs}$ $(P<0.05)$. The plasma levels of IL- $1 \beta$ and IL-18 in patients with ACHBLF were significantly higher than in patients with $\mathrm{CHB}$ and $\mathrm{HCs}(P<0.05)$. The relative mRNA level of NLRP3 in surviving patients decreased significantly compared with that in patients who did not survive after glucocorticoid treatment $(P<0.05)$. In conclusion, NLRP3 increased in patients with ACHBLF. Glucocorticoid could downregulate the expression of NLRP3 in surviving patients with ACHBLF.
\end{abstract}

Key words: ACHBLF, NLRP3, glucocorticoid treatment

\section{Introduction}

Because of its devastating effect on liver function, hepatitis B virus (HBV) poses a serious threat to the health of humans; approximately 360 million people around the world have been found to be positive for hepatitis B surface antigen (HBsAg) [1]. A variety of liver diseases such as hepatocellular cirrhosis, hepatocellular carcinoma, and acute-onchronic hepatitis B liver failure (ACHBLF) could possibly occur with the development of chronic HBV infections [2, 3]. Among those liver disorders, ACHBLF is considered to be the most serious because of the acute deterioration of liver function, multiorgan failure, and high mortality rates of $50 \%$ to $90 \%$ [4-6]. It has been estimated that up to 0.12 million people in Asia die of ACHBLF annually [7]. Liver transplantation is the primary rescue therapy strategy thus far, but it is highly limited by scarcity of liver donors and other reasons such as medical expense [8, 9]. Developing effective therapy methods while minimising the dependence of liver transplantation on the external liver source would be a better strategy to deal with ACHBLF. Nevertheless, the unclear pathogenesis of ACHBLF highly restricted the advancement of new treatment methods. It is thus 
important, yet challenging, to provide insight into the mechanism of ACHBLF development.

According to the primary pathological mechanism, dysregulated immunity by HBV infection and cell-mediated lymphocytotoxicity reaction result in an imbalance of proinflammatory cytokines which leads to the development of ACHBLF [10, 11]. NLRP3 is related to innate immunity and can produce proinflammatory cytokines via caspase-1[12]. Inflammasomes are cytosolic sensors that detect pathogen infections, tissue damage, or metabolic imbalances, and are helpful in maturation and release of several proinflammatory cytokines. The NLRs family consists of NLRs family pyrin domain containing 1(NLRP1), NLRs family pyrin domain containing 3(NLRP3), and NLRs family CARD domain containing 4 (NLRC4). NLRP3 and absent in melanoma 2 (AIM2)-like receptors are basic components of inflammasomes $[13,14]$. NLRP3 is the best characterised inflammasome that can recognise a range of substances including pathogen-associated molecular patterns, danger associated molecular patterns, and environmental irritants [15]. NLRP3 inflammasome activation contributes to the maturation of caspase-1, which further cleaves pro-interleukin (IL)-1 $\beta$ and pro-IL-18 into mature forms $[16,17]$. Studies in recent years reveal that NLRP3 plays a pivotal role in liver cirrhosis development [13] and it has a relationship with proliferation and metastasis of hepatocellular carcinoma [18]. Its activation also results in hepatocyte pyrotosis and the accelerated progression of NAFLD to NASH $[10,15]$.

In this study, we aimed to investigate NLRP3 messenger RNA (mRNA) expression in peripheral blood mononuclear cells (PBMCs) from patients with
ACHBLF and explore the potential effect of glucocorticoid on NLRP3 in this disease.

\section{Materials and Methods}

\section{Study population}

This study consisted of 124 patients admitted to Qilu Hospital of Shandong University May 2016 through December 2017. There were 70 patients in whom ACHBLF was diagnosed, 30 patients with $\mathrm{CHB}$, and 24 healthy volunteers as controls. Patients with $\mathrm{CHB}$ received their diagnosis based on being positive for HBsAg for at least 6 months. According to the Asian Pacific Association for the Study of the Liver [19], patients with ACHBLF were identified with the following characteristics: (a) CHB history; (b) progressive jaundice (TBIL $85 \mathrm{mmol} / \mathrm{L}$ ); (c) increasing international normalised ratio (INR) of 1.5 or decreasing prothrombin activity (PTA) to $40 \%$; and (d) onset of ascites and/or hepatic encephalopathy (HE) within 4 weeks. Exclusive criteria included co-infection with human immunodeficiency virus; hepatitis $\mathrm{C}$ virus; and hepatitis $\mathrm{D}$ virus; autoimmune liver diseases; metabolic liver disease; severe alcohol abuse; pregnancy; and liver tumours. The flowchart of selection of participants is shown in Figure 1.

Before this study, no patients had used corticosteroids for more than 6 months. Conservative treatment including nutritional support, disinfection and isolation especially oral care, reduction of aminotransferase, hepatoprotective medicines that can promote the regeneration and the repair of damaged hepatocytes and protect or mitigate injury to hepatocytes was provided to patients with ACHBLF. Before the glucocorticoid treatment, 39 patients received antiviral therapy and 31 patients did

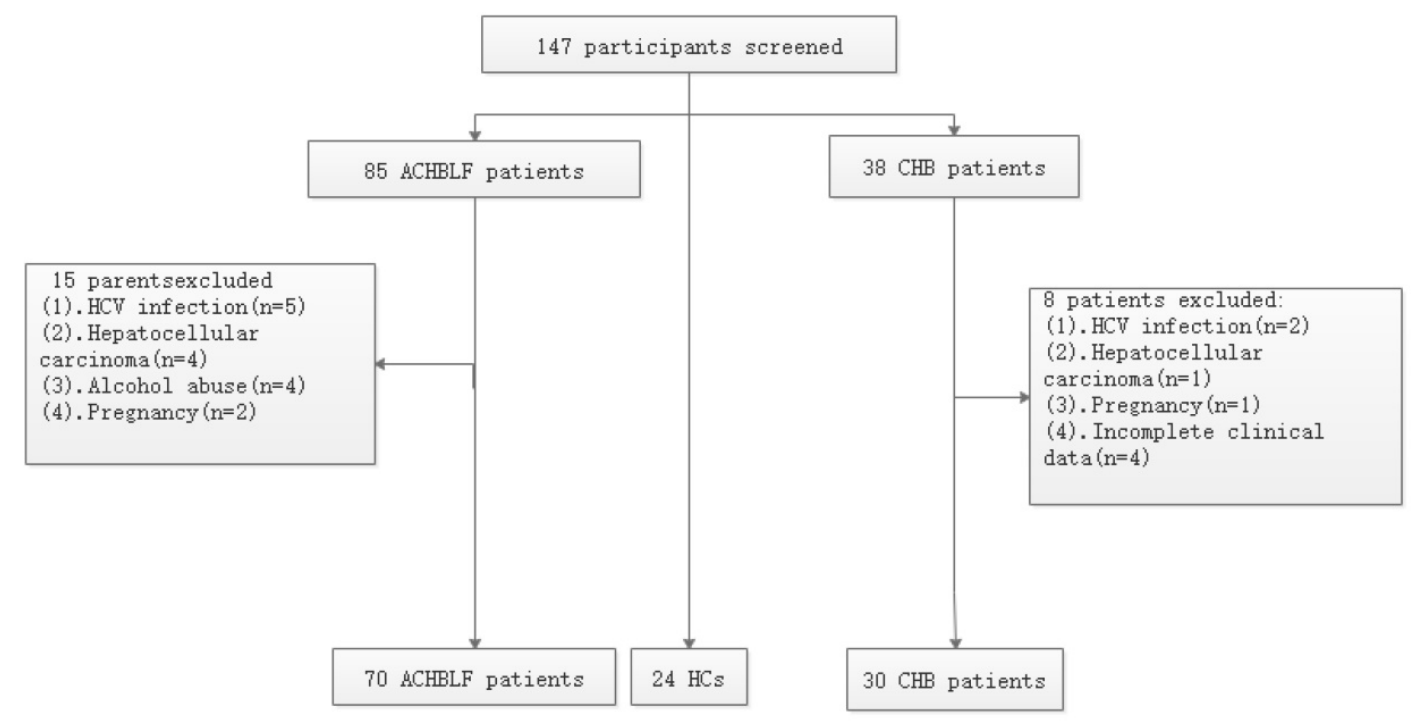

Figure 1. Flowchart depicting the participants' selection process. 
not. Of the 39 patients undergoing antiviral treatment, 25 underwent entecavir treatment at a dose of $0.5 \mathrm{mg}$ daily and 14 patients received $10 \mathrm{mg}$ of adefovir per day. In addition, of the 39 patients, 3, 9, 11, and 16 underwent antiviral treatment for 1 to 6 months, 6 to 12 months, 12 to 24 months, and more than 24 months, respectively. After ACHBLF was diagnosed, nucleoside analogues such as adefovir and entecavir were administered to patients to inhibit viral replication when serum HBV-DNA level was more than $10^{4}$ copy/mL[19]. Fifty-six patients underwent antiviral treatment. Among the 56 patients, 36 received entecavir treatment at a dose of $0.5 \mathrm{mg}$ daily and 20 patients were administered $10 \mathrm{mg}$ of adefovir per day.

After the diagnosis of ACHBLF was made, patients received the following 28-day prescriptions [20]: if treated with methylprednisolone (MSPL), it was given at $1 \mathrm{mg} / \mathrm{kg} /$ day (average: $80 \mathrm{mg} /$ day) for the first 3 days. MSPL $0.75 \mathrm{mg} / \mathrm{kg} /$ day (average: 60 $\mathrm{mg} /$ day) was given for the second 3 days. MSPL 0.5 $\mathrm{mg} / \mathrm{kg}$ /day (average: $40 \mathrm{mg} /$ day) was given for the third 3 days. If treated with prednisolone (PSL), it was given at $0.75 \mathrm{mg} / \mathrm{kg} /$ day (average: $60 \mathrm{mg} /$ day) for the first 3 days. PSL $0.5 \mathrm{mg} / \mathrm{kg} /$ day (average: 40 $\mathrm{mg}$ / day) was given for the second 3 days. PSL 0.25 $\mathrm{mg} / \mathrm{kg}$ / day (average: $20 \mathrm{mg} /$ day) was given for the third 3 days. Next, the glucocorticoid doses were reduced by 5 or $10 \mathrm{mg}$ at least every 4 days until the end of the 28th day for a complete withdrawal. The 3-month follow-up was set as the onset of the diagnosis of ACHBLF. The outcome was defined as death or survival at the end of the 3-month follow up. This study was approved by the local Ethical Committee of Qilu Hospital of Shandong University, and was conducted according the Declaration of Helsinki [21].

\section{RNA and complementary DNA preparation from PBMC}

Five $\mathrm{mL}$ of peripheral blood was obtained from all patients. PBMCs were collected after density gradient centrifugation with Ficoll-Paque Plus (GE Healthcare, Uppsala, Sweden). PBMCs were then washed three times with phosphate buffered saline. Total RNA of PBMCs was extracted by TRIzol (Invitrogen, Carlsbad, CA, USA). Two micrograms of RNA were reverse transcribed into complementary DNA (cDNA) using a first-strand cDNA synthesis kit (Thermo Fisher Scientific, Waltham, MA, USA).

\section{Real-time PCR}

Reverse-transcribed cDNA were subsequently subjected to real-time PCR analysis using a CFX96 ${ }^{\mathrm{TM}}$ Real-time System (Bio-Rad) with a SYBR Premix Ex TaqII kit (Takara). The PCR protocol was $95^{\circ} \mathrm{C}$ for $30 \mathrm{~s}$, followed by 35 cycles of $95^{\circ} \mathrm{C}$ for $5 \mathrm{~s}, 60^{\circ} \mathrm{C}$ for $30 \mathrm{~s}$, and the last step of $72^{\circ} \mathrm{C}$ for $30 \mathrm{~s}$. The primers were shown as follows: NLRP1-Forward: 5'-CCAGTTTGTGCGAA TCCA-3'; NLRP1-Reverse: 5'-CCAACGTAGAACTCC GAGAA-3'; NLRP3-Forward: 5'-TGAACAGCCACCT CACTT-3'; NLRP3-Reverse: 5'-CAACCACAATCTCC GAAT-3'; NLRC4-Forward: 5'-CAATAGCCGAGCCC TTAT-3'; NLRC4-Reverse: 5'-AGCCAAATCGTCCAA GTC-3'; caspase-1-Forward: 5'-CCGTTCCATGGGTG AAGGTA-3'; caspase-1-Reverse: 5'-ACGTGCTGTCA GAGGTCTTG-3'; IL-1 $\beta$-Forward: 5'-ACAGTGGCAA TGAGGATG-3'; IL-1 $\beta$-Reverse: 5'-TGTAGTGGTGGT CGGAGA-3'; IL-18-Forward: 5'-ATAGCCAGCCTAG AGGTA-3'; IL-18-Reverse: 5'-ATCAGGAGGATTCAT TTC-3'; $\beta$-Actin-Forward: 5'-AGTTGCGTTACACCCT TTCTTG-3'; $\beta$-Actin-Reverse: 5'-CACCTTCACCGTT CCAGTTTT-3'.

\section{Enzyme-linked immunosorbent assay}

Human IL-1 $\beta$ and IL-18 enzyme-linked immunosorbent assay (ELISA) kit (4 A Biotech Co., Ltd, Beijing, China) were used to detect IL-1 $\beta$ and IL-18 in plasma according to the manufacturer's instructions. The results were measured at $450 \mathrm{~nm}$ within 10 minutes using an enzyme-labelled instrument (Awareness USA).

\section{Western blot analysis}

Total protein was extracted from PBMCs. Briefly, the protein $(30 \mu \mathrm{g} /$ lane) was separated in $10 \%$ sodium dodecyl sulphate polyacrylamide gel and then transferred to a poly (vinylidene fluoride) membrane. NLRP3 (Cat No. TA336883), caspase-1, and IL-1 $\beta$ were detected by responding rabbit immunoglobulin $\mathrm{G}$ antibody (1:1000; Abcam). The results were visualised by the enhanced chemiluminescence system (Amersham, Arlington Heights, IL, USA).

\section{Clinical characteristics collection}

Alanine aminotransferase (ALT), aspartate aminotransferase (AST), albumin (ALB) and creatinine $(\mathrm{Cr})$, and total bilirubin (TBIL) were measured with COBAS integra 800 (Roche Diagnostics, Basel, Switzerland). HBsAg and hepatitis B e antigen (HBeAg) were measured through an electrochemiluminescence assay (Roche Diagnostics Ltd, Mannheim, Germany). HBV DNA was assayed by ABI 7300 PCR System (Applied Biosystems, Foster City, CA, USA). PTA and prothrombin time-international normalised ratio (PT-INR) were detected using ACL TOP 700 (Instrument Laboratory, Lexington, MA, USA). In addition, a model for end-stage liver disease (MELD) score was calculated according to the following original formula:

MELD score $=3.78^{*} \mathrm{LN}($ bilirubin $[\mathrm{mg} / \mathrm{dL}])+11.2^{*} \mathrm{LN}$ 
$(\mathrm{INR})+9.57$ * $\mathrm{LN}($ creatinine $[\mathrm{mg} / \mathrm{dL}])+6.43$

(aetiology: 0 if cholestatic or alcoholic; 1 otherwise).

\section{Statistical analysis}

Quantitative variables were expressed as mean \pm standard deviation; categorical values were expressed as number (percentage). Differences in quantitative variables were analysed using Mann-Whitney $U$ and Kruskal-Wallis tests. Differences in categorical variables were analysed using chi-square test. Spearman correlation was used to evaluate the correlation between variables and the diagnostic value of NLRP3. Statistical analyses were performed with SPSS 21.0 (SPSS, Chicago, IL, USA). All statistical tests were two-sided and value of $P<0.05$ was considered to be statistically significant.

\section{Results}

\section{Basic characteristics}

Data on mRNA expression levels of target genes were collected from 70 participants with ACHBLF before and after treatment, 30 patients with $\mathrm{CHB}$, and 24 healthy controls. The basic demographic and clinical characteristics of the 124 participants are listed in Table 1. There was no significant relationship between ACHBLF and $\mathrm{CHB}$ with respect to $\mathrm{HBeAg}$ $(P=0.979$; Mann-Whitney $U$ test), HBV DNA (+/-) $(P=0.196$; Mann-Whitney $U$ test), HBV DNA (log copies/ $\mathrm{ml})(P=0.719$; Mann-Whitney $U$ test $)$. There was also no significant difference between ACHBLF, $\mathrm{CHB}$, and $\mathrm{HCs}$ in regard to sex $(P=0.088$; Kruskal-Wallis $\mathrm{H}$ test), age $(P=0.058$; Kruskal-Wallis $\mathrm{H}$ test). However, there was an obvious difference among ACHBLF, CHB, and HCs with respect to ALT, AST, Cr, TBIL, INR, PTA $(\%)$, ALB $(P<0.001$; respectively, Kruskal- Wallis $\mathrm{H}$ test). Of the 70 patients with ACHBLF, 59 (84.29\%) had liver cirrhosis and $11(15.71 \%)$ did not.

\section{Expression of inflammation genes in different groups}

To investigate which molecular group of NLRs plays an important role in ACHBLF, we examined the relative mRNA expression levels in the PBMCs. Mean relative mRNA levels of NLRP1 in patients with ACHBLF, patients with $\mathrm{CHB}$, and healthy control patients were $0.220 \pm 0.429,0.112 \pm 0.192$, and $0.158 \pm$ 0.102 , respectively. No clear differences between these three groups were found $(P>0.05)$. Similarly, the mean relative mRNA levels of NLRC4 also show comparable results $(P>0.05)$ in patients with ACHBLF $(0.453 \pm$ $0.567)$, or CHB (0.302 \pm 0.512$)$, and healthy controls $(0.662 \pm 1.133)$. In contrast, the mRNA level of NLRP3 in patients with ACHBLF $(1.34 \pm 0.98)$ is significantly higher than those of the other two groups as shown in Figure 2A-C (patients with $\mathrm{CHB}$ and $\mathrm{HCs}$ ), whereas the HCs present a minimum level of $0.230 \pm 0.223$ $(P<0.01)$ compared to the $0.837 \pm 0.716$ of $\mathrm{CHB}$ group $(P=0.013)$.

Table 1. Baseline characteristics of the individuals enrolled in the study

\begin{tabular}{lllll}
\hline Variables & ACHBLF(n=70) & CHB(n=30) & HCs(n=24) & $P$ \\
\hline Age (year) & $50.40 \pm 12.64$ & $46.23 \pm 11.87$ & $44.63 \pm 5.396$ & $0.058 \dagger$ \\
Sex (male/female) & $47 / 23$ & $22 / 8$ & $11 / 13$ & $0.088 \dagger$ \\
TBIL (mmol/L) & $327.2 \pm 153.3$ & $42.98 \pm 47.82$ & $11.36 \pm 3.778$ & $0.000 \dagger$ \\
ALT (U/L) & $358.9 \pm 431.6$ & $111 \pm 106.7$ & $16.83 \pm 7.631$ & $0.000 \dagger$ \\
AST (U/L) & $263.5 \pm 262.5$ & $63 \pm 45.9$ & $16.29 \pm 6.524$ & $0.000 \dagger$ \\
INR & $2.44 \pm 0.71$ & $1.168 \pm 0.109$ & $1.088 \pm 0.099$ & $0.000 \dagger$ \\
PTA (\%) & $33.8 \pm 7.15$ & $79.97 \pm 10.15$ & $100.6 \pm 15.92$ & $0.000 \dagger$ \\
ALB (g/L) & $38 \pm 6.324$ & $40.33 \pm 4.042$ & $48.78 \pm 2.216$ & $0.000 \dagger$ \\
Cr & $59.87 \pm 7.97$ & $53.37 \pm 12.13$ & $53.54 \pm 7.15$ & $0.000 \dagger$ \\
HBeAg (+/-) & $24 / 46$ & $10 / 20$ & NA & $0.979 \ddagger$ \\
HBV DNA (+/-) & $37 / 33$ & $16 / 14$ & NA & $0.196 \ddagger$ \\
HBV DNA (log & $4.82 \pm 1.297$ & $4.681 \pm 1.413$ & NA & $0.719 \ddagger$ \\
copies/mL) & & & & \\
MELD score & $19.73 \pm 5.168$ & NA & NA & \\
Ascites & 56 & 0 & 0 & \\
Encephalopathy & 22 & 0 & 0 & \\
Liver cirrhosis & 59 & 0 & 0 & $0.931 \ddagger$ \\
Antiviral treatment $(+/-)$ & $39 / 31$ & $17 / 13$ & 0 &
\end{tabular}

Data were expressed as mean \pm standard deviation. TBIL, total bilirubin; ALT, Alanine aminotransferase; AST, aspartate aminotransferase; INR, international normalised ratio; PTA, prothrombin time activity; ALB, albumin; $\mathrm{Cr}$, creatinine; $\mathrm{HBeAg}$, hepatitis B e antigen; MELD, model for end-stage liver disease; NA, not available; ACHBLF, acute-on-chronic hepatitis B liver failure; $\mathrm{CHB}$, chronic hepatitis B; HCs, healthy controls. †: Kruskal-Wallis H test ‡: Mann-Whitney $U$ test

\section{Expression of NLRP3-related cytokines and genes}

Because NLRP3 was shown to be involved in ACHBLF, we tried to further study its related cytokines and genes. The plasma levels of IL-1 $\beta$ and IL-18 were measured by ELISA. Both cytokines increased significantly in patients with ACHBLF $(\mathrm{IL}-1 \beta 3722 \pm 2346$ pg/L, IL-18 $524.9 \pm 90.6$ pg/L) compared with those of patients with $\mathrm{CHB}$ (IL-1 $\beta$ $2140 \pm 1158 \mathrm{pg} / \mathrm{L}, \mathrm{IL}-18365.7 \pm 62.79 \mathrm{pg} / \mathrm{L}$, both $P<0.01)$ and HCs (IL-1 $1160 \pm 962.6$ pg/L, IL-18 $280 \pm$ $68.63 \mathrm{pg} / \mathrm{L}$, both $P<0.01)$. We also observed that levels of IL-1 $\beta$ and IL-18 in patients with CHB are higher than those of HCs (both $P<0.01$, Figure $2 \mathrm{D}$ and E). The mRNA levels of caspase-1, IL-1 $\beta$, and IL-18 in patients with ACHBLF (caspase-1 $0.942 \pm 0.633$, IL-1 $\beta$ $1.342 \pm 0.759$ and IL-18 $0.065 \pm 0.084)$ were more than those of patients with CHB (caspase- $10.449 \pm 0.398$, $P<0.01$; IL-1 $0.916 \pm 0.476 P<0.01$ and IL-18 $0.030 \pm$ $0.034 P<0.01$ ) and in HCs (caspase-1 $0.451 \pm 0.291$, $P<0.01$; IL-1 $\beta 0.538 \pm 0.460 P<0.01$ and IL-18 $0.006 \pm$ $0.012 P<0.01$; Figure $2 \mathrm{~F}-\mathrm{H})$. The results further confirm that NLRP3 and related cytokines/genes caspase-1, IL-1 $\beta$, and IL-18 were involved in the progression of ACHBLF. 


\section{Correlation between NLRP3 mRNA expression and clinicopathologic features}

Then, we analysed the correlation between NLPR3 mRNA levels and clinical parameters using Spearman analysis. We found that the expression of NLRP3 mRNA was significantly positively associated with TBIL ( $\mathrm{r}=0.290, P=0.015)$, and MELD $(\mathrm{r}=0.268$, $P=0.025$; Figure $3 \mathrm{~A}, \mathrm{~B})$, and negatively correlated with ALB $(\mathrm{r}=-0.311, P=0.009)$ and PTA $(\mathrm{r}=-0.332, P$ $=0.005$; Figure $3 \mathrm{D}, \mathrm{E})$. There was no correlation between NLRP3 mRNA level, and $\mathrm{Cr}(\mathrm{r}=0.168, P=$ $0.165)$, ALT $(r=0.091, P=0.454)$, and AST $(r=0.178, P$
A

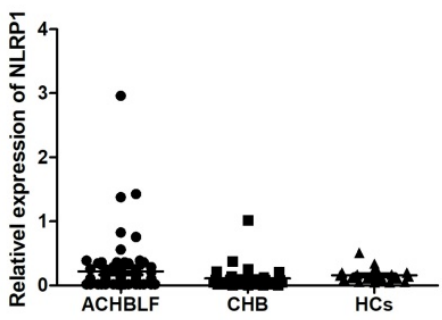

C

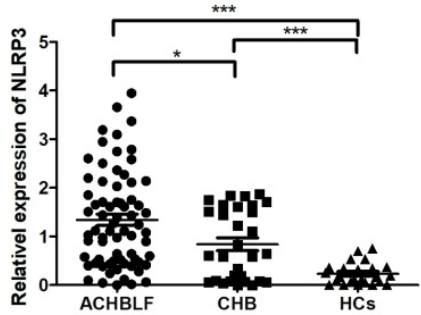

E

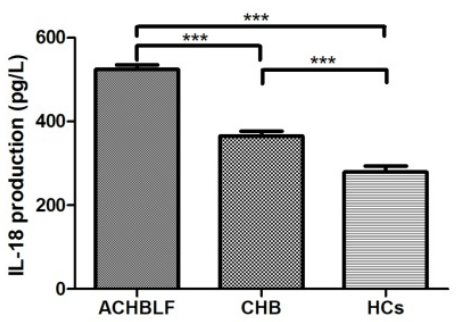

G

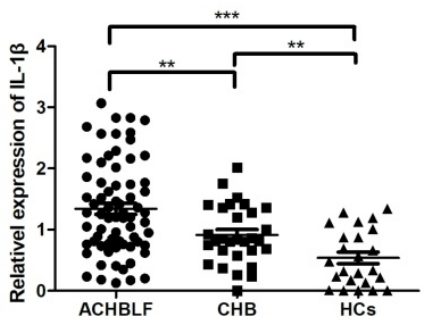

B

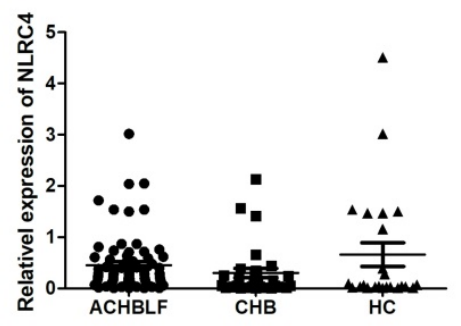

D

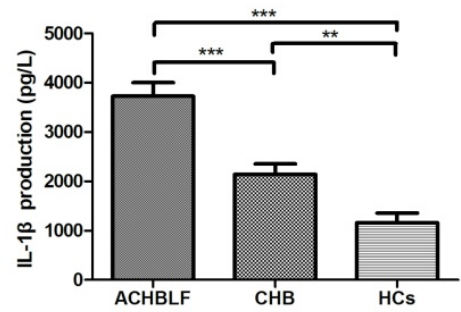

$\mathbf{F}$

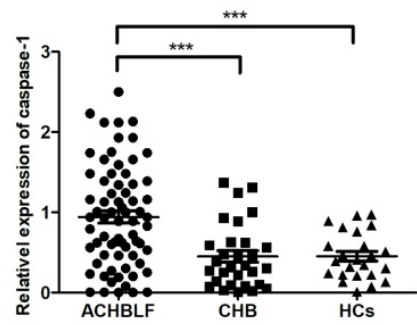

$\mathbf{H}$

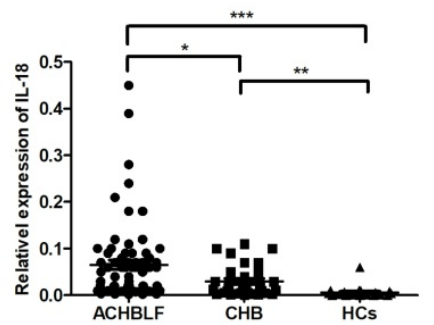

Figure 2. The comparison of NLRPI, NLRP3, and NLRC4 mRNA levels in PMBC among the three participant groups; $A$ : the comparison of NLRPI in patients with $A C H B L F, C H B$, and HCs; $B$ : the comparison of NLRC4 in patients with ACHBLF, CHB, and HCs; C: the comparison of NLRP3 in patients with ACHBLF, $\mathrm{CHB}$, and HCs. $D$, E: the comparison of serum IL- $1 \beta$ and IL-18 levels in patients with ACHBLF, CHB, and HCs; F, G: the comparison of IL- $1 \beta$ and IL-18 mRNA levels in PBMC among patients with ACHBLF, CHB, and $\mathrm{HCs}$; $\mathrm{H}$ : the comparison of caspase-1 mRNA level in PBMC among patients with ACHBLF, CHB, and HCs. ACHBLF: acute-on-chronic hepatitis B liver failure; CHB: chronic hepatitis B; HCs: healthy controls.

\section{NLRP3 and related cytokines/genes decreased in surviving patients after glucocorticoid}

At the end of the 90-day follow-up period, 38 patients with ACHBLF survived after 28 days of patients $(1.512 \pm 1.105)$ and non-survivors $(1.136 \pm 0.777, P=0.111)$. After 7-day treatment, surviving patients $(0.555 \pm 0.646)$ still had similar expression of NLRP3 with non-survivors $(0.907 \pm 0.998, P=0.08)$. However, with 28-day treatment, surviving patients $(0.514 \pm 0.460)$ had higher expression of NLRP3 than non-survivors $(1.296 \pm 1.034, P<0.01$; Figure 4A).

There is no clear difference in caspase-1, IL-1 $\beta$, and IL-18 mRNA levels between surviving patients (caspase-1 $1.029 \pm 0.722$, IL-1 $\beta 1.424 \pm$ 0.764 and IL-18 $0.079 \pm 0.109)$ and non-survivors (caspase-1 $0.899 \pm 0.510$, $P=0.398$; IL-1 $\beta 1.243 \pm 0.752, P=0.324$; IL-18 $0.048 \pm 0.033, P=0.123)$. On the seventh day of treatment, the mRNA levels of caspase-1, IL-1 $\beta$, and IL-18 of surviving patients (caspase- $10.647 \pm$ 0.520 , IL-1 $1.059 \pm 0.496$ and IL-18 $0.018 \pm 0.019)$ were lower than those of non-survivors (caspase-1 $1.029 \pm 0.759$, $P<0.01$; IL-1 $\beta 1.568 \pm 0.582, \quad P<0.01$; IL-18 $0.057 \pm 0.078, P<0.01)$. This trend was retained on the 28th day of treatment: mRNA levels of caspase-1, IL-1 $\beta$, and IL-18 were lower in patients who survived (caspase-1 $0.467 \pm 0.305$, IL-1 $\beta 0.690 \pm 0.417$ and IL-18 $0.007 \pm$ $0.007)$ than in those who did not (caspase-1 $1.005 \pm 0.698, P<0.01$; IL-1 $\beta$ $1.651 \pm 0.500, P<0.01$; IL-18 $0.071 \pm 0.070$, $P<0.01$; Figure 4B-D). Using western blot analysis, we also detected the protein levels of NLRP3, caspase-1, cleaved caspase- 1 , pro-IL-1 $\beta$, and IL-1 $\beta$ in patients who survived. As shown in Figure 4G, with the treatment of glucocorticoid, the activation of caspase-1 and IL-1 $\beta$ production in 
patients who survived was decreased. Glucocorticoid could alter mortality by decreasing the expression of NLRP3.

\section{Glucocorticoid effect on liver function in patients with ACHBLF}

TBIL and PTA, as the major laboratory indices, were used to assess patients with ACHBLF. Before treatment, there was no difference in TBIL and PTA between patients who survived (TBIL $307.2 \pm 164.4$; PTA $34.63 \pm 7.111$ ) and those who did not (TBIL 350.9 $\pm 137.8, P=0.238$; PTA $32.81 \pm 7.195, P=0.293)$. On the 7 th and 28th days of treatment, TBIL decreased and PTA increased in patients who survived $(P<0.05)$, but both remained the same in those who did not survive $(P>0.05)$ (Figure 4E, F).

\section{Discussion}

In our study, we first investigated the NLRs mRNA level in PMBC from 70 patients with ACHBLF, 30 patients with $\mathrm{CHB}$, and $24 \mathrm{HCs}$ to determine which molecular patterns take part in the progression of ACHBLF. Our data showed that NLRP3 increased significantly. Then, we measured the NLRP3 and related cytokines/genes. Our results show that the NLRP3 mRNA level in patients with ACHBLF is higher than in patients with $\mathrm{CHB}$ and in HCs (Figure 2). The trend was also observed in caspase-1, IL-1 $\beta$, and IL-18. All the aforementioned results suggested that NLRP3 may be involved in the development of ACHBLF. According to the Spearman analysis, NLRP3 mRNA level in PBMCs from patients with ACHBLF is found to be positively correlated with TBIL and MELD, while negatively correlated with PTA and ALB (Figure 3). These results indicate that NLRP3 might be associated with the severity of ACHBLF.

There are many reasons that ACHBLF occurs. The reactivation of HBV was considered the leading cause of ACHBLF [19]. Patients with ACHBLF caused by hepatotoxic drugs and herbal medicines account for a large number in the Asia-Pacific region [22, 23]. Alcohol abuse was also a major cause of ACHBLF [24]. Various types of infections could contribute to liver failure, including bacterial, parasitic, fungal, spirochetal, protozoal, and helminthic in patients with CHB [19]. ACHBLF is regarded as the most serious liver impairment because of its acute deterioration of liver functions, multi-organ failure and high mortality of 50-90\% [4-6]. Glucocorticoid can prevent hepatocytes from necrosis and progressive acute deterioration by protecting cell membrane, lysosomal enzymes, and mitochondria [11, 25] . Glucocorticoid can rapidly inhibit excess immune response and inflammatory reaction, and has been confirmed to be effective in early stage of ACLF [26]. 
A

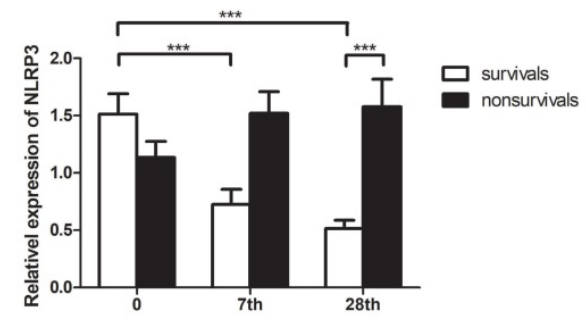

C

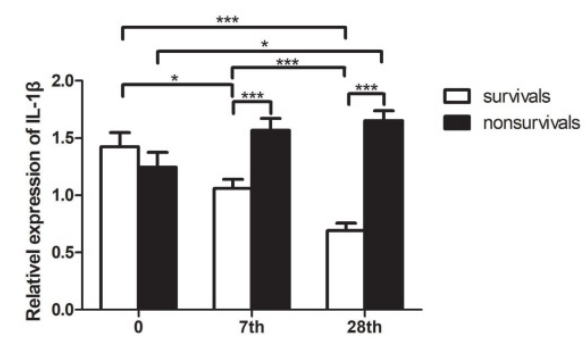

E

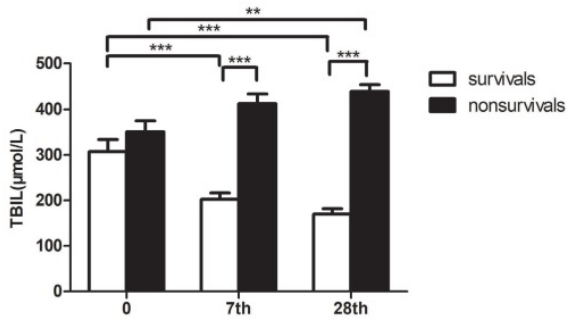

B

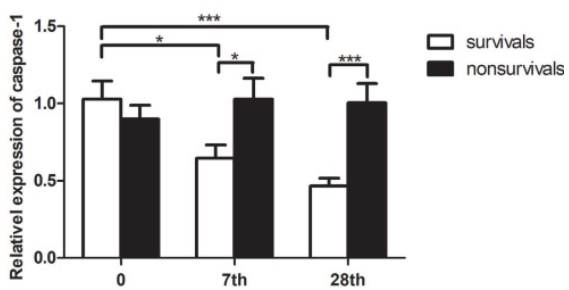

D

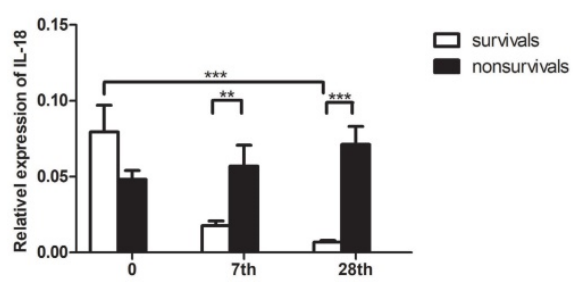

F

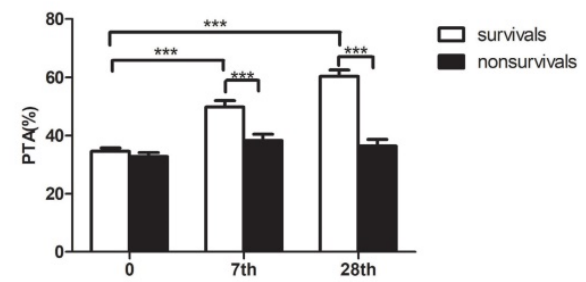

G

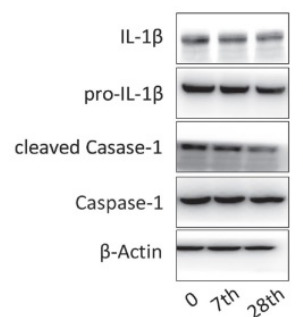

Figure 4. Effect of glucocorticoid on patients with ACHBLF. A, B, C, D: The comparison of NLRP3, caspase-1, IL- $1 \beta$ and IL-18 mRNA levels in patients who survived and those who did not before and after glucocorticoid treatment; E, F: The comparison of TBIL and PTA in patients who survived and those who did not before and after glucocorticoid treatment; G: the NLRP3, caspase-1, cleaved caspase-1, pro-IL-1 $\beta$, and IL-1 $\beta$ protein levels of patients who survived before and after glucocorticoid treatment.

NLRP3 inflammasome is a large intracellular multiprotein complex that consists of an inflammasome sensor molecule (NLR) and adaptor proteins, such as apoptosis-associated speck-like protein containing a caspase-recruitment domain (ASC) and the precursor procaspase-1[27]. NLRP3 was the best characterised member of the NLR family, and it is critical in regulating innate immune response. NLRP3 inflammasome activation leads to the maturation of caspase- 1 , which further cleaves
pro-IL-1 $\beta$ and pro-IL-18 into mature forms. In our study, the mRNA level of NLRP3 of ACHBLF patients was higher than that of patients with $\mathrm{CHB}$ and HCs. NLRP3 downstream gene caspase-1 was also activated, and this is consistent with NLRP3-caspase-1 inflammasome as a critical inflammatory mediator propelling the host response against infection, injury, and disease [28]. The expression of proinflammatory cytokines increased significantly under liver failure [29], and we found the mRNA level of IL-1 $\beta$ and IL-18 
in patients with ACHBLF was higher than that of $\mathrm{CHB}$ and HCs in this study. The result was also confirmed by the plasma level measured through ELISA. TBIL and PTA are often used to judge patients with ACHBLF, and we found that the NLRP3 mRNA level of these patients was positively correlated with TBIL and negatively correlated with PTA. All results indicate that NLRP3 makes a contribution to the progression of ACHBLF and is related to disease severity. Both dynamic changes of NLRP3 and related cytokines/ genes decreased gradually in patients who survived during glucocorticoid treatment in our survey. These results demonstrate that glucocorticoid can decrease IL-1 $\beta$ and IL-18 by downregulating NLRP3.

There are some limitations to this study. First, the number of patients with ACHBLF who received 28 days of glucocorticoid treatment is relatively small. We believe that the validation of our findings would be performed in larger, multicentred, prospective cohorts before its clinical usage. Second, coagulopathy and high bleeding risk made it unfeasible to obtain liver biopsy specimens from patients with liver failure. So it is impossible to detect the NLRP3 expression in liver tissue directly. Third, NLRP3 was also considered to lead to pyroptosis, which was responsible for liver failure; its precise mechanism of pathogenesis remains to be determined [30].

In summary, our results demonstrated that the expression level of NLRP3 is increased and positively correlated with disease severity in patients with ACHBLF. The findings suggest that NLRP3 may take part in the pathogenesis of ACHBLF disease. Our results also showed that the expression of NLRP3 decreased gradually after glucocorticoid treatment in patients who survived. The findings further indicate NLRP3 may play a role in pathogenesis of ACHBLF disease.

\section{Acknowledgments}

This study was funded by grants from the key project of Chinese Ministry of Science and Technology (2018ZX10301406-005 and 2017ZX10202202).

\section{Authorship}

Guarantor of the article: Dr Kai Wang accepts full responsibility for the conduction of the study. He had full access to the data and control of the decision to publish.

Author contributions: Qian Zhao: study design, analysis and interpretation of the data, statistical analysis; approved final manuscript draft submitted. Chen-Si Wu, Yu Fang, Yu Qian, He Wang: acquisition of data, study support; approved final manuscript draft submitted. Yu-Chen Fan: analysis and interpret- ation of data, critical revision of manuscript; approved final manuscript draft submitted. Kai Wang: study design, critical revision of the manuscript, study supervision; approved final manuscript draft submitted.

\section{Competing Interests}

The authors have declared that no competing interest exists.

\section{References}

1. Akhtar F, Rehman S: A Public Health Analysis on Gaps in Disease Monitoring and Opportunities for Improved Care for the Management of Hepatitis B and C. Cureus 2018, 10(1):e2077.

2. Sun FK, Gao S, Fan YC, Shi CH, Zhang ZH, Wang LY, Li F, Li XY, Ji XF, Zhao J et al: High promoter methylation levels of glutathione-S-transferase M3 predict poor prognosis of acute-on-chronic hepatitis B liver failure. Hepatol Res 2017, 47(6):566-573.

3. Zhong Y, Guo Y, Liu X, Zhang J, Ma T, Shu J, Yang J, Zhang J, Jia Z, Li Z: Serum Glycopatterns as Novel Potential Biomarkers for Diagnosis of Acute-on-Chronic Hepatitis B Liver Failure. Sci Rep 2017, 7:45957.

4. Gao S, Ji XF, Li F, Sun FK, Zhao J, Fan YC, Wang K: Aberrant DNA methylation of G-protein-coupled bile acid receptor Gpbar1 predicts prognosis of acute-on-chronic hepatitis B liver failure. J Viral Hepat 2015, 22(2):112-119.

5. Jalan R, Gines P, Olson JC, Mookerjee RP, Moreau R, Garcia-Tsao G, Arroyo V, Kamath PS: Acute-on chronic liver failure. J Hepatol 2012, 57(6):1336-1348.

6. Zhou N, Wang K, Fang S, Zhao X, Huang T, Chen H, Yan F, Tang Y, Zhou H, Zhu J: Discovery of a Potential Plasma Protein Biomarker Panel for Acute-on-Chronic Liver Failure Induced by Hepatitis B Virus. Front Physiol 2017, 8:1009.

7. Gao S, Huan SL, Han LY, Li F, Ji XF, Li XY, Fan YC, Wang K: Overexpression of serum sST2 is associated with poor prognosis in acute-on-chronic hepatitis B liver failure. Clin Res Hepatol Gastroenterol 2015, 39(3):315-323.

8. Fan YC, Wang N, Sun YY, Xiao XY, Wang K: TIPE2 mRNA Level in PBMCs Serves as a Novel Biomarker for Predicting Short-Term Mortality of Acute-on-Chronic Hepatitis B Liver Failure: A Prospective Single-Center Study. Medicine (Baltimore) 2015, 94(39):e1638.

9. Lin S, Chen J, Wang MF, Han LF, Zhang HY, Dong J, Zeng DW, Jiang JJ, Zhu YY: Prognostic nomogram for acute-on-chronic hepatitis B liver failure. Oncotarget 2017, 8(65):109772-109782.

10. Wan X, Xu C, Yu C, Li Y: Role of NLRP3 Inflammasome in the Progression of NAFLD to NASH. Can J Gastroenterol Hepatol 2016, 2016:6489012.

11. Zhang JJ, Fan YC, Zhao ZH, Yang Y, Dou CY, Gao S, Wang K: Prognoses of patients with acute-on-chronic hepatitis B liver failure are closely associated with altered SOCS1 mRNA expression and cytokine production following glucocorticoid treatment. Cell Mol Immunol 2014, 11(4):396-404.

12. Wei $\mathrm{O}, \mathrm{Mu} \mathrm{K}$, Li T, Zhang $\mathrm{Y}$, Yang Z, Jia X, Zhao W, Huai W, Guo P, Han L: Deregulation of the NLRP3 inflammasome in hepatic parenchymal cells during liver cancer progression. Lab Invest 2014, 94(1):52-62.

13. Alegre F, Pelegrin P, Feldstein AE: Inflammasomes in Liver Fibrosis. Semin Liver Dis 2017, 37(2):119-127.

14. Donnelly MC, Hayes PC, Simpson KJ: Role of inflammation and infection in the pathogenesis of human acute liver failure: Clinical implications for monitoring and therapy. World I Gastroenterol 2016, 22(26):5958-5970.

15. Lu Y, Xu S, Chen H, He M, Deng Y, Cao Z, Pi H, Chen C, Li M, Ma Q et al: $\mathrm{CdSe} / \mathrm{ZnS}$ quantum dots induce hepatocyte pyroptosis and liver inflammation via NLRP3 inflammasome activation. Biomaterials 2016, 90:27-39.

16. Jo EK, Kim JK, Shin DM, Sasakawa C: Molecular mechanisms regulating NLRP3 inflammasome activation. Cell Mol Immunol 2016, 13(2):148-159.

17. Li X, Yan X, Wang Y, Wang J, Zhou F, Wang H, Xie W, Kong H: NLRP3 inflammasome inhibition attenuates silica-induced epithelial to mesenchymal transition (EMT) in human bronchial epithelial cells. Exp Cell Res 2018, 362(2):489-497.

18. Fan SH, Wang YY, Lu J, Zheng YL, Wu DM, Li MO, Hu B, Zhang ZF, Cheng $\mathrm{W}$, Shan Q: Luteoloside suppresses proliferation and metastasis of hepatocellular carcinoma cells by inhibition of NLRP3 inflammasome. PLOS One 2014, 9(2):e89961.

19. Sarin SK, Kumar A, Almeida JA, Chawla YK, Fan ST, Garg H, de Silva HJ, Hamid SS, Jalan R, Komolmit P et al: Acute-on-chronic liver failure: consensus recommendations of the Asian Pacific Association for the study of the liver (APASL). Hepatol Int 2009, 3(1):269-282.

20. Zhao J, Zhang JY, Yu HW, He YL, Zhao JJ, Li J, Zhu YK, Yao QW, Wang JH, Liu HX et al: Improved survival ratios correlate with myeloid dendritic cell restoration in acute-on-chronic liver failure patients receiving methylprednisolone therapy. Cell Mol Immunol 2012, 9(5):417-422.

21. Hellmann F, Verdi M, Schlemper BR, Jr., Caponi S: 50th anniversary of the Declaration of Helsinki: the double standard was introduced. Arch Med Res 2014, 45(7):600-601. 
22. Lee KH, Lee MK, Sutedja DS, Lim SG: Outcome from molecular adsorbent recycling system (MARS) liver dialysis following drug-induced liver failure. Liver Int 2005, 25(5):973-977.

23. Mattei A, Rucay P, Samuel D, Feray C, Reynes M, Bismuth H: Liver transplantation for severe acute liver failure after herbal medicine (Teucrium polium) administration. J Hepatol 1995, 22(5):597.

24. Moreau R, Arroyo V: Acute-on-chronic liver failure: a new clinical entity. Clin Gastroenterol Hepatol 2015, 13(5):836-841.

25. Fujiwara K, Yasui S, Yonemitsu Y, Fukai K, Arai M, Imazeki F, Suzuki A, Suzuki H, Sadahiro T, Oda S et al: Efficacy of combination therapy of antiviral and immunosuppressive drugs for the treatment of severe acute exacerbation of chronic hepatitis B. J Gastroenterol 2008, 43(9):711-719.

26. Mao Q, Zhang HY, You JP, Zhang XQ: Severe acute exacerbation of chronic hepatitis $\mathrm{B}$ during pegylated interferon treatment and early intervention with corticosteroid. Virol J 2012, 9:136.

27. Ratsimandresy RA, Dorfleutner A, Stehlik C: An Update on PYRIN Domain-Containing Pattern Recognition Receptors: From Immunity to Pathology. Front Immunol 2013, 4:440.

28. Gross O, Yazdi AS, Thomas CJ, Masin M, Heinz LX, Guarda G, Quadroni M, Drexler SK, Tschopp J: Inflammasome activators induce interleukin-1alpha secretion via distinct pathways with differential requirement for the protease function of caspase-1. Immunity 2012, 36(3):388-400.

29. Wang J, Ren H, Yuan X, Ma H, Shi X, Ding Y: Interleukin-10 secreted by mesenchymal stem cells attenuates acute liver failure through inhibiting pyroptosis. Hepatol Res 2018, 48(3):E194-E202.

30. Wree A, Eguchi A, McGeough MD, Pena CA, Johnson CD, Canbay A, Hoffman HM, Feldstein AE: NLRP3 inflammasome activation results in hepatocyte pyroptosis, liver inflammation, and fibrosis in mice. Hepatology 2014, 59(3):898-910. 